\title{
Supplementary material for the article: Academic performance of students from entrance to graduation via quasi U-statistics: a study at a Brazilian research university
}

Rafael Pimentel Maia, Hildete Prisco Pinheiro*and Aluísio Pinheiro

Department of Statistics, University of Campinas, Brazil

Appendix A. Analytical results of the MLE for the normal and triangular distribution

For the Normal distribution

Let

$$
\mu_{g}=E\left[X_{i}^{g}\right], \quad \mu_{g 2}=E\left[\left(X_{i}^{g}\right)^{2}\right], \quad \mu_{g 3}=E\left[\left(X_{i}^{g}\right)^{3}\right] \text { and } \mu_{g 4}=E\left[\left(X_{i}^{g}\right)^{4}\right]
$$

be the first, second, third and fourth moments of $X_{i}^{g}$. Then,

$$
\begin{aligned}
\phi_{1}\left(x_{1}^{g}\right) & =E\left[\phi\left(X_{1}^{g}, X_{2}^{g}\right) \mid X_{1}^{g}=x_{1}^{g}\right]=\left(x_{1}^{g}\right)^{2}-2 x_{1}^{g} \mu_{g}+\mu_{g 2}, \\
E\left[\phi_{1}\left(X_{1}^{g}\right)\right] & =E\left[\left(X_{1}^{g}\right)^{2}-2 X_{1}^{g} \mu_{g}+\mu_{g 2}\right]=2 \sigma_{g}^{2}, \\
E\left(\phi_{1}^{2}\right) & =\mu_{g 4}-4 \mu_{g} \mu_{g 3}+3 \mu_{g 2}^{2} .
\end{aligned}
$$

Therefore,

$$
\sqrt{n_{g}}\left(\bar{D}_{g}-2 \sigma^{2}\right) \stackrel{D}{\rightarrow} N\left(0,4 \xi_{1}\right)
$$

where

$$
\xi_{1}=E\left[\phi_{1}\left(X_{1}^{g}\right)-\theta_{g}\right]=\mu_{g 4}-4 \mu_{g} \mu_{g 3}+3 \mu_{g 2}^{2}-4 \sigma_{g}^{4} .
$$

*Corresponding author: E-mail address: hildete@ime.unicamp.br 
For $\bar{D}_{g g^{\prime}}$, which is a U-statistic of degree $(1,1)$, let

$$
\begin{aligned}
\theta_{g g^{\prime}} & =E\left(\phi\left(X_{1}^{g}, X_{2}^{g^{\prime}}\right)\right)=\mu_{g 2}-2 \mu_{g} \mu_{g^{\prime}}+\mu_{g^{\prime} 2}, \\
\phi_{10} & =E\left[\phi\left(X_{1}^{g}, X_{2}^{g^{\prime}}\right) \mid X_{1}^{g}=x_{1}^{g}\right]=x_{1}^{g}-2 x_{1}^{g} \mu_{g^{\prime}}+\mu_{g^{\prime} 2}, \\
\phi_{01} & =E\left[\phi\left(X_{1}^{g}, X_{2}^{g^{\prime}}\right) \mid X_{2}^{g^{\prime}}=x_{2}^{g^{\prime}}\right]=\mu_{g 2}-x_{2}^{g^{\prime}} \mu_{g}+x_{2}^{g^{\prime}} .
\end{aligned}
$$

Then,

$\xi_{10}=E\left[\phi_{10}\left(X_{1}^{g}\right)-\theta_{g g^{\prime}}^{2}\right]=\mu_{g 4}-4 \mu_{g 3} \mu_{g^{\prime}}+2 \mu_{g 2} \mu_{g^{\prime} 2}-4 \mu_{g} \mu_{g^{\prime}} \mu_{g^{\prime} 2}+\mu_{g^{\prime} 2}^{2}-\theta_{g g^{\prime}}^{2}$

and

$\xi_{01}=E\left[\phi_{01}\left(X_{2}^{g^{\prime}}\right)-\theta_{g g^{\prime}}^{2}\right]=\mu_{g^{\prime} 4}-4 \mu_{g^{\prime} 3} \mu_{g}+2 \mu_{g^{\prime} 2} \mu_{g 2}-4 \mu_{g} \mu_{g^{\prime}} \mu_{g 2}+\mu_{g 2}^{2}-\theta_{g g^{\prime}}^{2}$.

Therefore,

$$
\operatorname{Var}\left(\bar{D}_{g g^{\prime}}\right)=\frac{1}{n_{g}} \xi_{10}+\frac{1}{n_{g^{\prime}}} \xi_{01}
$$

As $B_{n}$ is a linear function of U-statistics $\left(\bar{D}_{g}\right.$ and $\left.\bar{D}_{g g^{\prime}}\right)$, its variance will be dependent on the moments of $X_{i}^{g}$.

For instance, if $G=2$,

$$
\operatorname{Var}\left(B_{n}\right)=\frac{n_{1}^{2} n_{2}^{2}}{n^{2}(n-1)^{2}} 4\left[\frac{\xi_{10}}{n_{1}}+\frac{\xi_{01}}{n_{2}}+\frac{\xi_{1}}{n_{1}}+\frac{\xi_{1}}{n_{2}}\right]+O\left(\frac{1}{n}\right)
$$

For the normal distribution we can apply the property of invariance of maximum likelihood estimators (MLE) to get the estimation of the mean and variance of $B_{n}$. Therefore, the MLE estimators of the mean and variance are

$$
\begin{aligned}
\hat{\mu} & =\bar{X}, \\
\hat{\sigma}^{2} & =(n-1) S^{2} / n .
\end{aligned}
$$

As $\hat{\sigma}^{2}$ is biased for $\sigma^{2}$, we will use $S^{2}$ as an estimator of $\sigma^{2}$, since it is an unbiased estimator for $\sigma^{2}$. The estimators of the second, third and fourth moments are

$$
\begin{aligned}
& \hat{\mu}_{2}=S^{2}+\bar{X}^{2}, \\
& \hat{\mu}_{3}=3 \bar{X} S^{2}+\bar{X}^{3}, \\
& \hat{\mu}_{4}=3 S^{4}+6 S^{2} \bar{X}^{2}+\bar{X}^{4} .
\end{aligned}
$$




\section{For the Triangular distribution}

Let $Z$ be a random variable with a triangular distribution limited in $[a, b]$ with mode $m$, then

$$
f(z)=\frac{2(z-a)}{(b-a)(m-a)} I(a \leq z \leq m)+\frac{2(b-z)}{(b-a)(b-m)} I(m \leq z \leq b) .
$$

The $k$-th moments of $Z$ are

$$
\begin{aligned}
\mu_{k} & =c_{1}\left(\frac{m^{k+2}}{k+2}-\frac{a m^{k+1}}{k+1}-\frac{a^{k+2}}{k+2}+\frac{a^{k+2}}{k+1}\right) \\
& +c_{2}\left(\frac{b^{k+2}}{k+1}-\frac{b^{k+2}}{k+2}-\frac{b m^{k+1}}{k+1}+\frac{m^{k+2}}{k+2}\right),
\end{aligned}
$$

for all $k=1,2, \ldots$, with $c_{1}=\frac{2}{(b-a)(m-a)}$ and $c_{2}=\frac{2}{(b-a)(b-m)}$.

If $Z_{1}, Z_{2}, \ldots, Z_{n}$ are independent random variables with p.d.f given by (A.3) and $\mathbf{Z}=\left(Z_{(1)}, Z_{(2)}, \ldots, Z_{(n)}\right)$ is the vector of order statistics, the distribution of $\mathbf{Z}$ is given by

$$
f\left(z_{(1)}, z_{(2)}, \ldots, z_{(n)}\right)=\left(\frac{2}{b-a}\right)^{n}\left\{\prod_{i=1}^{r} \frac{z_{(i)}-a}{m-a} \prod_{i=r+1}^{n} \frac{b-z_{(i)}}{b-m}\right\},(
$$

where $r$ is such that $Z_{(r)} \leq m<z_{(r+1)}, z_{(0)} \equiv a$ and $z_{(n+1)} \equiv b$.

Therefore, for fixed values of $a$ and $b$, satisfying $a<z_{(1)}$ and $b>z_{(n)}$, we have that

$$
\max _{a \leq m \leq b} L(a, m, b \mid \mathbf{z})=\left(\frac{2}{b-a}\right)^{n}\{M(a, b, \hat{r}(a, b))\},
$$

where

$\hat{r}(a, b)=\arg \max _{r \in\{1, \ldots, n\}} M(a, b, r)$ and $M(a, b, r)=\prod_{i=1}^{r-1} \frac{z_{(i)}-a}{z_{(r)}-a} \prod_{i=r+1}^{n} \frac{b-z_{(i)}}{b-z_{(r)}}$.

The MLE of $m$ (as a function of $a$ and $b$ ) is given by $\hat{m}(a, b)=Z_{(\hat{r}(a, b))}$. Note that the function $\hat{r}(a, b)$ indicates in which order statistic the MLE of $m$ is achieved as a function of the lower and upper limit, $a$ and $b$, respectively.

From (A.6) we have

$$
\max _{S(a, m, b)}[\log \{L(a, m . b ; \mathbf{z})\}]=\max _{a<z_{(1)}, b>z_{(n)}}[\log \{n \log 2+G(a, b)\}],
$$

where $S(a, b)=\left\{(a, m, b) \mid a<z_{(1)}, b>z_{(n)}, a \leq m \leq b\right\}$ and $G(a, b)=$ $\log \{M(a, b, \hat{r}(a, b))\}-n \log (b-a)$. 
Note that $G(a, b)$ is defined only for values of $a<z_{(1)}$ and $b>z_{(n)}$. So, the three dimensional problem of maximization of $L(a, m, b ; \mathbf{z})$ is reduced to a two dimensional problem of maximization of $G(a, b)$ under $a<z_{(1)}$ and $b>z_{(n)}$. From the likelihood structure, one can see that for all values of $m$ such that $z_{(1)}<m<z_{(n)}$, the likelihood $L(a, m, b ; \mathbf{z}) \rightarrow 0$ (and therefore $\log L(a, m, b ; \mathbf{z}) \rightarrow \infty)$ when $a \uparrow z_{(1)}$ or $b \downarrow z_{(n)}$.

To obtain the MLE for the triangular distribution, Kotz and Dorp (2004) suggests the use of the routines BSearch and ABSearch combined. The MLE in the triangular case can be obtained using the software MLE-Estimator (www.seas.gwu.edu/dorpjr/tab4/publications_book.html).

\section{Appendix B. Algorithm for the quasi U-statistic nonparametric method}

Step 1 - Compute the relative gain according to the introduction.

Step 2 - Separate the data set according to the groups of interest.

Step 3 - Compute observed $\bar{D}_{g}$ 's, $\bar{D}_{g g^{\prime}}$ 's and then $B_{n}$ from the data, call it $B_{n}(o b s)$.

Step 4 - Apply the jackknife re-sampling method to obtain the SE of $B_{n}$.

Step 4.1 - Compute the jackknife's replication $B_{n}(-i)$ by removing from the sample the $i-t h$ individual and then apply Step 3 again, for all individuals in the sample, one by one.

Step 4.2 - Compute the $B_{n}(\cdot)=\sum_{i=1}^{n} \frac{B_{n}(-i)}{n}$.

Step 4.3 - The Jackknife Standard Error $\left(S E_{J}\right)$ is then estimated by

$$
S E_{J}=\left[\frac{n-1}{n} \sum_{i=1}^{n}\left[B_{n}(-i)-B_{n}(\cdot)\right]^{2}\right]^{1 / 2} .
$$

Step 5 - Compute the $p$-value (one-sided test), (i.e, $P\left[B_{n}>B_{n}(\right.$ obs $\left.)\right]$ ), using the normal approximation below for $B_{n}$ (see detaiis in the main file of the article) and Standard Deviation given by the $S E_{J}$ calculated in step 4.

$$
\left(M_{n} V_{n}^{*}\right)^{-1 / 2} B_{n} \stackrel{\mathcal{D}}{\longrightarrow} N(0,1) \text { as } n \rightarrow \infty,
$$

with $M_{n}=O\left(n^{2}\right), V_{n}^{*}=U_{n}^{(2,2)}-U_{n}^{(3)}, U_{n}^{(2,2)}=\left(\begin{array}{c}n \\ 2\end{array}\right)^{-1} \sum_{1 \leq i<j \leq n} \phi^{2}\left(X_{i}, X_{j}\right)$ and $U_{n}^{(3)}=\frac{1}{n(n-1)(n-2)} \sum_{i \neq i \neq l} \phi\left(X_{i}, X_{j}\right) \phi\left(X_{i}, X_{l}\right)$. 


\section{References}

Kotz, S. and Dorp, J. R. van (2004) Beyond Beta, other continuous families of distributions with bounded support and applications. World Scientific Press, Singapure. 